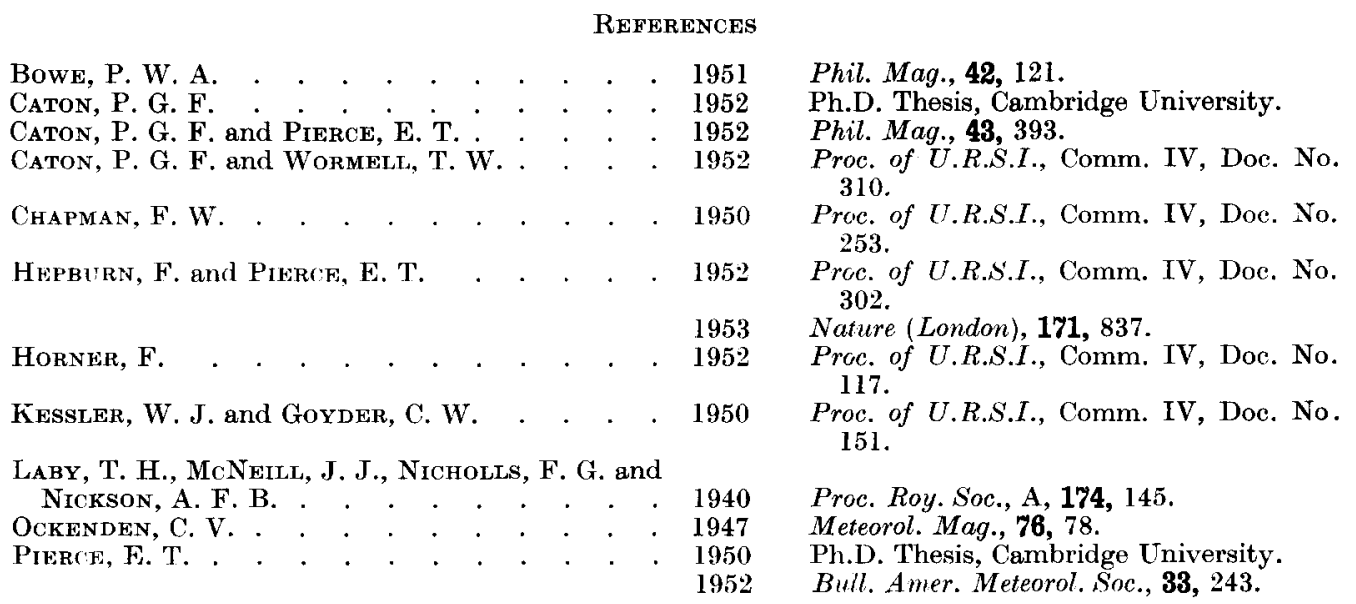

\title{
The Abundance of Atmospheric Carbon Dioxide and its Isotopes
}

\author{
LEO GOLDBERG \\ The Observatory of the University of Michigan, Ann Arbor, Michigan, U.S.A.
}

\section{INTRODUCTION}

Analysis of the absorption spectrum of the Earth's atmosphere is best accomplished when the Sun is used as a light source. The chief advantages of this method are: (1) the enormous brightness of the Sun, which favours the use of high-resolution spectrometers; and (2) the availability of a very long atmospheric path, which can vary between $8 \mathrm{~km}$ and $300 \mathrm{~km}$ N.T.P., depending upon the altitude of the Sun. For several reasons, however, accurate spectroscopic determinations of the abundances of atmospheric constituents have not been made until recently. The chief difficulties have been: (1) limitations in resolving power imposed by inefficient gratings and insensitive infra-red energy detectors; (2) absence of data on absolute absorption coefficients and true line widths; and (3) lack of an adequate theory of line formation in a non-homogeneous atmosphere.

All of these difficulties have now been satisfactorily overcome. The development of improved gratings and infra-red detectors has made possible the recording of relatively faint molecular lines for which the total absorption is independent of the true line width. Hence, when sufficiently small concentrations of absorbing gas are employed in the laboratory, the line absorption coefficients may be determined by direct proportionality with the measured equivalent widths (GoLdBERG, MoHLER, Donovan, 1952). The atmospheric abundances may then be derived from similar measurements in the solar spectrum. WILSON and WEJLS (1946) have also shown how the integrated absorption coefficient of a vibration-rotation band may be obtained from laboratory measurements with spectroscopes of low resolving power. The necessary formulae relating the individual line absorption coefficients to the 
band absorption coefficient have been given by Crawford and Dinsmore (1950), Penner and Weber (1951), and Eggers and Crawford (1951).

The problem of the interpretation of the absorption-line intensities when the density and temperature vary with altitude has been treated by vas DE HuLsT (1945), and later by the writer (1951). It has been shown, for example, that the equivalent widths of molecular lines of low rotational energy are nearly identical with those that would be formed in an isothermal atmosphere of uniform density. The effective temperature defined by the Boltzmann equation is about 20 per cent lower than the ground value, while the true line width, produced chiefly by collisions, is about onehalf the ground value. Expressions have been derived for the relatively small deviations from a Boltzmann distribution and for the slow increase of collisional line width with excitation potential. An important consequence of the theoretical results is that the true widths of atmospheric lines may now be deduced from curve of growth experiments in the laboratory (LOCKE and HERzBERG, 1953). The broadening effect of one atmosphere of air upon the absorbing molecules is determined for any given line and the corresponding atmospheric line width is then calculated from theory. By this method, abundances may also be derived from the equivalent widths of very strong lines.

These modern methods have been applied to the atmospheric spectra of oxygen (van de Hulst, 1945), methane (Goldberg, 1951; Nielsen and Migeotte, 1952), and carbon monoxide (Locke and Herzberg, 1953; Howard and Shaw, 1952). The abundance of carbon dioxide, which has not previously been determined spectroscopically, is of special interest both because of its importance to the life cycle and because of the occurrence in the infra-red solar spectrum of bands arising from the two isotopic forms $\mathrm{C}^{13} \mathrm{O}_{2}{ }^{16}$ and $\mathrm{C}^{12} \mathrm{O}^{16} \mathrm{O}^{18}$. According to J. S. Haldane (1936), the carbon dioxide content of country air, from chemical measurements, varies between the limits 0.021 and 0.044 per cent. A diurnal variation in the measurements, presumably due to photosynthesis, was quite definitely noted, as were the effects of exhalation from the soil and of combustion in the City of London. The present investigation had three aims: (1) to check the chemical determinations of the carbon dioxide content by the spectroscopic method; (2) to investigate possible seasonal variations in the $\mathrm{CO}_{2}$ content; and (3) to determine the abundances of the isotopic varieties.

The observations of the carbon dioxide bands were made with the high-dispersion spectrometer and Cashman PbS cell that is attached to the Snow telescope of the Mount Wilson Observatory and is operated under a co-operative arrangement by the staff of the McMath-Hulbert Observatory of the University of Michigan. Two main groups of $\mathrm{CO}_{2}$ bands are found in the region $1 \cdot 5 \mu-2 \cdot 5 \mu$; three strong bands occur at $2 \mu$ and four weaker ones in the $1 \cdot 6 \mu$ region. The strength of the $2 \mu$ bands makes them unsuitable for analysis, both because of uncertainties in the line widths and because of the overlapping of neighbouring line wings. On the other hand, the band centre at $1.60 \mu$ contains several unblended lines of intermediate and low intensity, and the wings do not appear to overlap seriously. The band is shown in Fig. 1 . For investigation of the abundances of $\mathrm{C}^{13}$ and $\mathrm{O}^{18}$, two bands were chosen-one due to $\mathrm{C}^{12} \mathrm{O}^{16} \mathrm{O}^{18}$ at $2 \cdot 04 \mu$ and the other arising from $\mathrm{C}^{13} \mathrm{O}_{2}{ }^{16}$ at $2 \cdot 05 \mu$ (see Fig. 5).

\section{Absorption Coefficients}

The first step in the abundance determination was the measurement of the line absorption coefficients in the laboratory. To a sufficient approximation, the integrated 
absorption coefficient per molecule in level $J$ for individual lines in parallel bands of linear triatomic molecules is given by EGGERs and CRAWFORD (1951) as

$$
S_{, J J^{\prime}}=S_{0} \frac{v}{v_{0}} \frac{|m|}{2 J+1},
$$

where $v$ is the frequency of the line and $\nu_{0}$ that of the band centre; $S_{0}$ is the integrated absorption coefficient of the entire vibration-rotation band per molecule in the ground vibrational state, and $m$ is the serial number of the line, which is equal to $J+1$ in the positive branch and to $-J$ in the negative branch. For the two isotope bands, it was assumed that the integrated absorption coefficient was identical with that for the corresponding band of $\mathrm{C}^{12} \mathrm{O}_{2}{ }^{16}$ at $2 \cdot 01 \mu$. According to WEBER, HoLm,

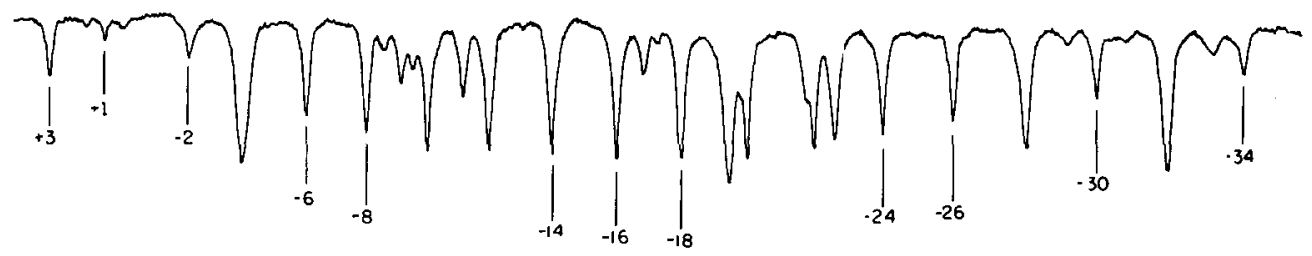

Fig. 1. A portion of the $1 \cdot 60 \mu$ band of $\mathrm{CO}_{2}$ in the solar spectrum. The serial numbers of the measured lines are indicated

and Penner (1952), its value is $1 \cdot 1 \times 10^{-9} \mathrm{~cm}^{2}-\mathrm{sec} .^{-1}$. For the $1.60 \mu$ band $S_{0}$ was determined from a series of absorption-cell tracings made in the laboratory by Dr. MoHLER and very kindly furnished by him. The source employed was a d.c. motionpicture arc with very high infra-red intensity (McMath and MohLer, 1949), the absorption being produced over a total path length of $295 \mathrm{~cm}$ as a result of two traversals of a steel absorption cell filled with a mixture of $\mathrm{CO}_{2}$ and air. Tracings were made with four different partial pressures of $\mathrm{CO}_{2}$, equal to $191,305,495$, and $846 \mathrm{~mm} \mathrm{Hg}$, but sufficient air was always introduced to maintain the total pressure constant at three atmospheres. It was anticipated that the pressure broadening would thus be great enough to insure linearity between the equivalent widths and the absorption coefficients.

The line absorption coefficient over the entire path length becomes

$$
\tau=N_{J} S_{. J J^{\prime}}=N_{J} S_{0} \frac{v}{v_{0}} \frac{|m|}{2 J+1},
$$

where $N_{J}$, the number of molecules per square centimetre along the absorbing path in level $J$, is given by the Boltzmann equation:

$$
N_{J}=\frac{N_{0}}{Q_{r}}(2 . J+1) e^{-E_{J} / k T} .
$$

It should be kept in mind that, in the symmetrical forms of carbon dioxide, e.g. $\mathrm{C}^{12} \mathrm{O}_{2}{ }^{16}, \mathrm{C}^{13} \mathrm{O}_{2}{ }^{16}$, only the levels of even $J$ are populated and therefore the line absorption coefficient given by equation (2) must be doubled. Equation (2), as it is written, is valid for $\mathrm{C}^{12} \mathrm{O}^{16} \mathrm{O}^{18}$, for which lines of both odd and even $J$ occur. In the Boltzmann 
formula, $N_{0}$ is the number of molecules per square centimetre in the ground vibrational state, which at room temperature is essentially equal to the total in all states, $E_{J}$ is the rotational energy and $Q_{r}$ is the rotational partition function. To a very high approximation (EgGers and Crawford, 1951),

$$
Q_{r}=0.695 \frac{T}{B_{0}},
$$

where the rotational constant $B_{0}=0.391$ kaysers.*

In equation (2) above, all quantities are known except $S_{0}$. If the logarithms of the equivalent widths, $A$ (in sec. ${ }^{-1}$ ), of the $\mathrm{CO}_{2}$ lines recorded in the laboratory are

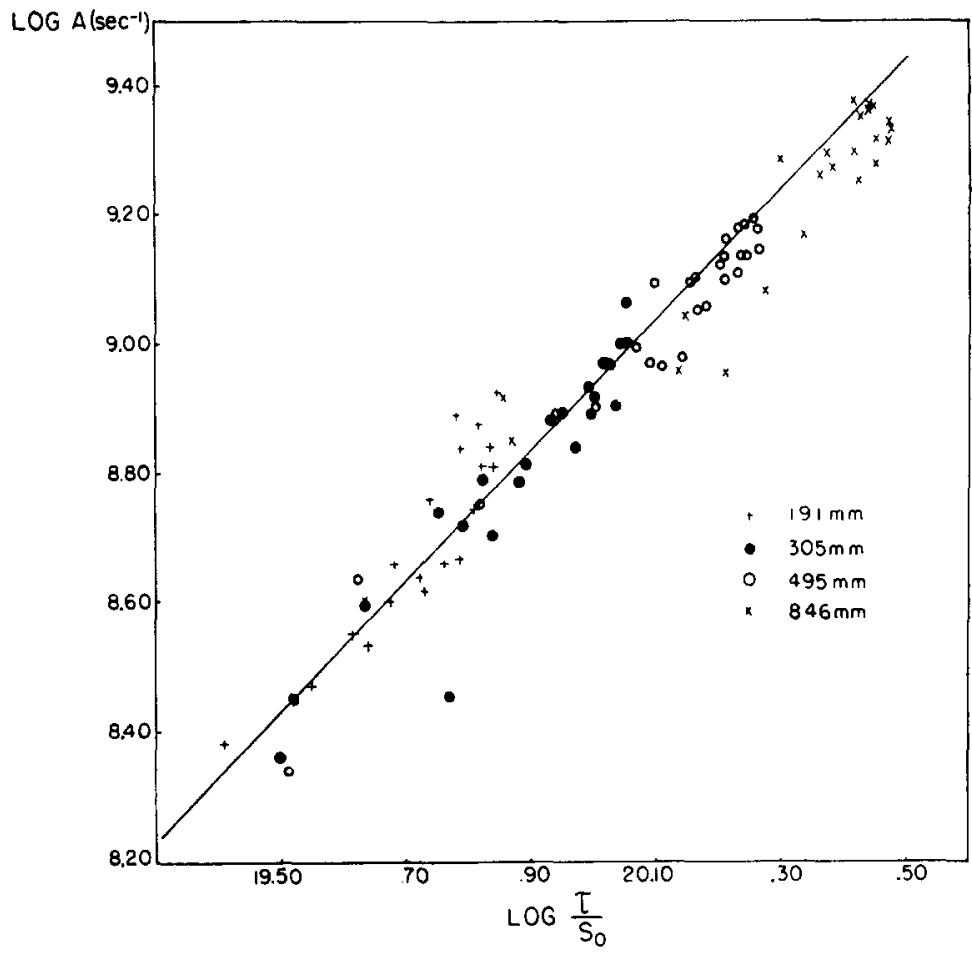

Fig. 2. Laboratory curve of growth for $\mathrm{CO}_{2}$

plotted against the logarithms of the absorption coefficients given by equation (2), the plotted points should define the curve of growth for $\mathrm{CO}_{2}$ lines. In equation (2), all quantities are known except $S_{0}$. In the linear branch of the curve of growth $A$ is equal to $\tau$. Hence, if $\log A$ is plotted against $\log \tau / S_{0}$, the intercept of the linear branch of the curve on the $\log A$ axis is $\log S_{0}$. The results are plotted in Fig. 2, where it may be seen first that there is satisfactory agreement among the observations made at different partial pressures of $\mathrm{CO}_{2}$, and second that most of the points fall on the linear portion of the curve of growth. The value of $S_{0}$ is $8 \cdot 6 \times 10^{-12} \mathrm{~cm}^{2}$-sec. ${ }^{-1}$.

$$
\text { 3. Abundance of } \mathrm{C}^{12} \mathrm{O}_{2}{ }^{16}
$$

The data for the abundance determinations consist of measurements of equivalent widths of lines in the $1.60 \mu$ band, observed in the solar spectrum on various dates

* The recently adopted unit for wave number, formerly denoted $\mathrm{em}^{-1}$, is the kayser, abbreviated $\mathrm{K}$. 
between 24th August, 1949, and 23rd January, 1953. The altitudes of the Sun at the times of observation were such that the number of air masses varied between 1 and about 8. If the integrated absorption coefficient for air mass unity is $\tau$, then its value when the Sun is at zenith distance $z$ is $\tau$ sec. $z$. We cannot assume that the equivalent widths will be equal to the line absorption coefficients without actually constructing the curve of growth for $\mathrm{CO}_{2}$ in the Earth's atmosphere. This was accomplished as follows. For each of twelve lines in both positive and negative branches, $\log A$ was plotted against $\log$ sec. $z$ from observations made on the dates of 19 th, 20th, 21st, and 28th December, 1950. The twelve sets of points were then superposed in the usual way to form a composite curve which was represented very well by a

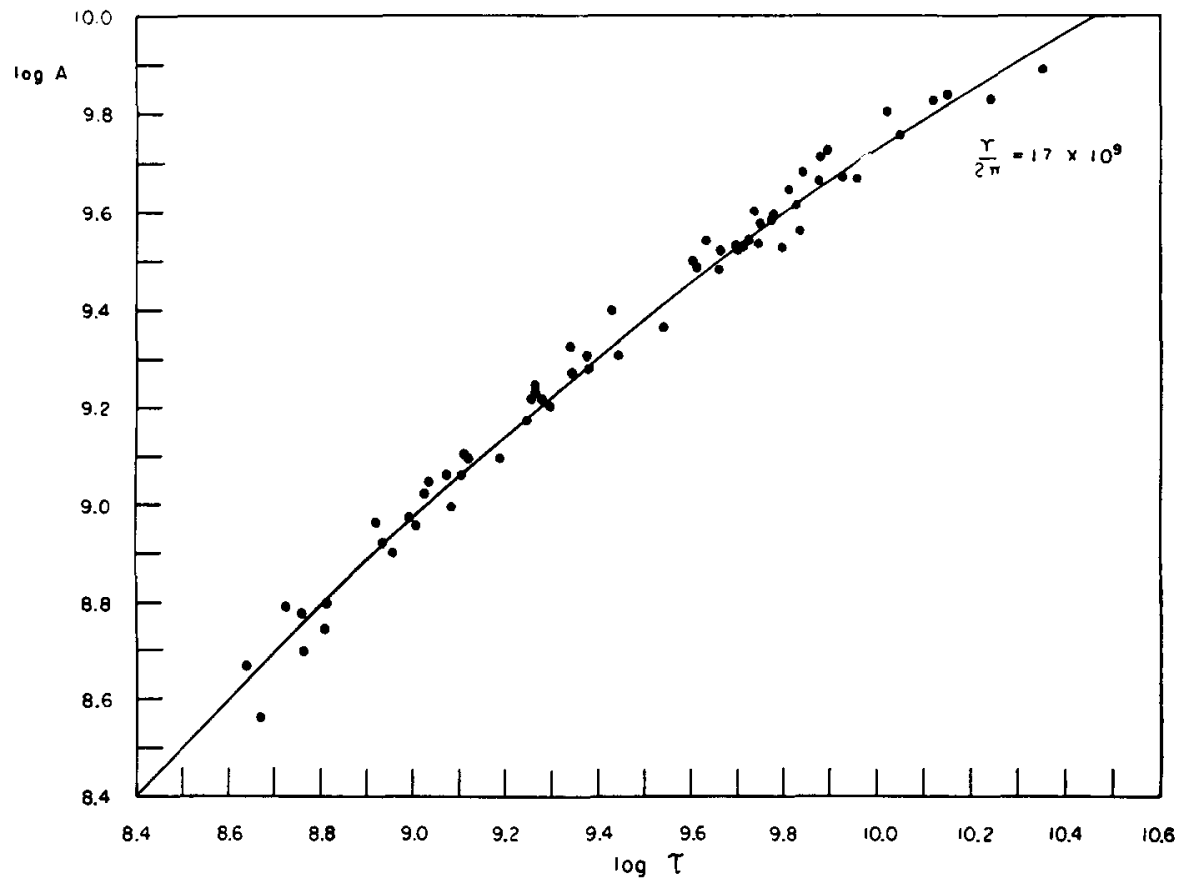

Fig. 3. The curve of growth for $\mathrm{CO}_{2}$ in the Earth's atmosphere

theoretical curve of growth for pure collisional broadening with a line width at halfintensity, $\gamma / 2 \pi$, equal to $1.7 \times 10^{9} \mathrm{sec}^{-1}$. This value is not precise inasmuch as most of the points are fairly close to the linear branch of the curve of growth. The theoretical curve and the empirical points are shown in Fig. 3.

The neglect of Doppler broadening as compared with collisional broadening in the Earth's atmosphere has already been justified by vaN DE HULST (1945). It is further illustrated by calculation of the parameter $a$, the ratio of the collisional width to the most probable Doppler displacement. The value of $\gamma / 2 \pi$ at ground level is twice the value derived from the curve of growth, or $3.4 \times 10^{9} \mathrm{sec}^{-1}$. On the other hand, the Doppler displacement for molecular weight 44 and a temperature of $300^{\circ} \mathrm{K}$ is $2 \cdot 1 \times 10^{8} \mathrm{sec}^{-1}$. The value of $a$ is therefore about 16 at ground level, although it will decrease at higher altitudes, where the contribution to the line intensity is relatively small.

The curve of growth in Fig. 3 was employed to convert all available values of $\log A$ to values of $\log \tau$. For dates other than those in December, 1950, the measurements 
were confined chiefly to negative branch lines, as shown in Fig. 1. If we define the quantity

$$
Y=\log \tau-\log S_{0} \frac{v}{\nu_{0}}|m|,
$$

then by equations (2) and (3),

$$
Y=\log \frac{N_{0}}{Q_{r}}-\frac{0 \cdot 244}{T} J(J+1),
$$

where $N_{0}$ is the number of molecules per square centimetre through the zenith.

It is obvious that if the values of $Y$ are plotted against $J(J+1)$, the slope and intercept of the resulting straight line yield the effective temperature and abundance,

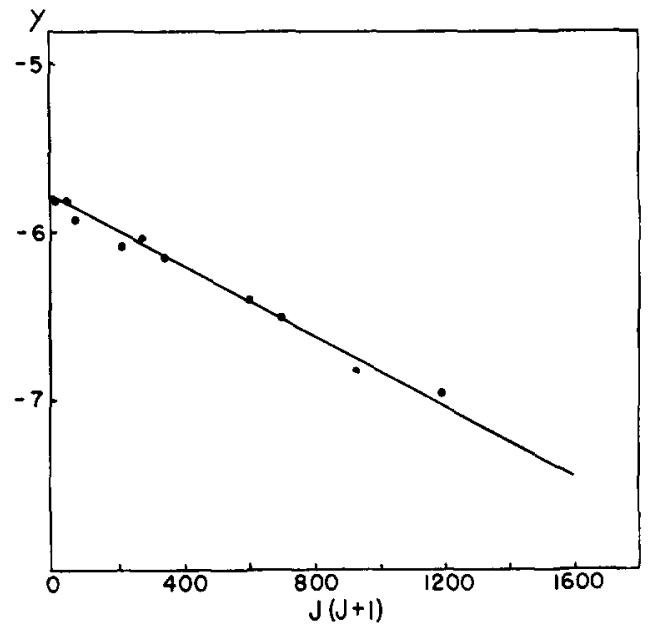

Fig. 4. Graphical determination of abundance of $\mathrm{CO}_{2}$ and effective temperature, 5th November, 1949

respectively. Fig. 4 shows a representative plot of $Y$ vs. $J(J+1)$ for the date of 5 th November, 1949. Table 1 contains the results of the separate temperature and abundance determinations. The uncertainty in the temperatures may be as high as 10 per cent and therefore probably masks any correlation with the ground temperatures given in column 4 . The mean values of the ground and effective temperatures are $290^{\circ}$ and $247^{\circ} \mathrm{K}$, respectively. The ratio of 1.18 represents good average agreement with theory.

Table 1. Abundance of $\mathrm{CO}_{2}$ and effective temperatures over Mount Wilson

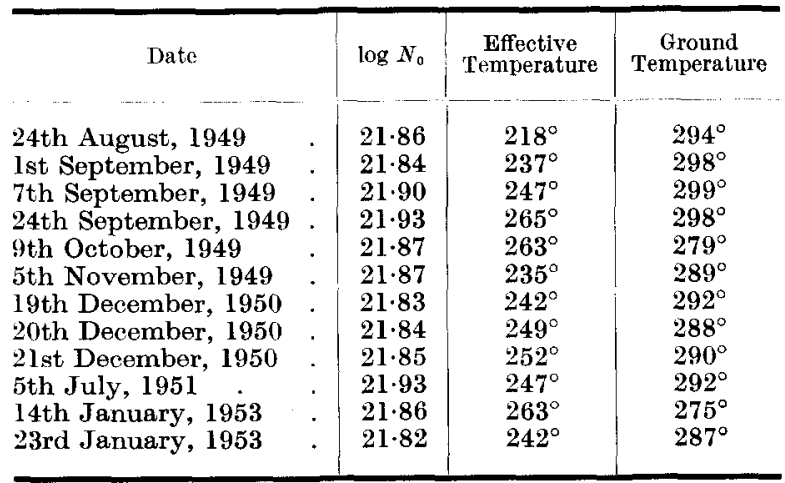


The average value of $\log N_{0}$ is 21.87 and the maximum variation exhibited in Table 1 is about \pm 12 per cent, which is of the same order of magnitude as the error of determination. It seems safe to conclude that the $\mathrm{CO}_{2}$ content over Mount Wilson remained constant within about 10 per cent during the twelve days listed in Table 2.

Table 2. Abundances of $\mathrm{C}^{13} \mathrm{O}_{2}{ }^{16}$ and $\mathrm{C}^{12} \mathrm{O}^{16} \mathrm{O}^{18}$ over Mount Wilson, December, 1950

\begin{tabular}{|c|c|c|c|c|c|}
\hline$\lambda(\AA)$ & $m_{\mathrm{C}^{13} \mathrm{O}_{2}{ }^{16}}$ & $m_{\mathrm{C}^{18} \mathrm{O}^{18} \mathrm{O}^{18}}$ & $\tau_{1}+\tau_{2}$ & $N_{v_{\mathrm{g}}}$ & $N_{0_{1}}$ \\
\hline $\begin{array}{l}20,428 \\
20,416 \\
20,411 \\
20,389 \\
20,374 \\
20,369 \\
20,351 \\
20,362 \\
20,348 \\
20,335 \\
20,323\end{array}$ & $\begin{array}{r}+9 \\
+13 \\
+15 \\
+23 \\
+29 \\
+31 \\
+39\end{array}$ & $\begin{array}{l}-14 \\
-10 \\
-\quad 9 \\
-\quad 2 \\
+\quad 3 \\
+\quad 5 \\
+\quad 11 \\
+\quad 7 \\
+\quad 12 \\
+17 \\
+22\end{array}$ & $\begin{array}{l}1.429 \times 10^{-7} \\
1.513 \times 10^{-7} \\
1.333 \times 10^{-7} \\
0.955 \times 10^{-7} \\
0.519 \times 10^{-7} \\
0.700 \times 10^{-7} \\
0.422 \times 10^{-7} \\
0.160 \times 10^{-7} \\
0.240 \times 10^{-7} \\
0.320 \times 10^{-7} \\
0.202 \times 10^{-7}\end{array}$ & $\begin{array}{l}1.375 \times 10^{19} \\
1.466 \times 10^{19} \\
1.880 \times 10^{19} \\
1.393 \times 10^{19}\end{array}$ & $\begin{array}{l}4.014 \times 10^{19} \\
3.700 \times 10^{19} \\
3.169 \times 10^{19} \\
3.298 \times 10^{19} \\
2.596 \times 10^{18} \\
4 \cdot 156 \times 10^{10} \\
3.843 \times 10^{18}\end{array}$ \\
\hline
\end{tabular}

There is no evidence for a seasonal variation of any magnitude. The average total number of molecules per square centimetre through the zenith is $7 \cdot 4 \times 10^{21}$. Corrected to sea level, the abundance in centimetres N.T.P. is 320 and if for the entire atmosphere the reduced height is $8.04 \times 10^{5} \mathrm{~cm}$, the percentage abundance is $0 \cdot 040$. This is in reasonably good agreement with chemical determinations (HaLdane, 1936).

\section{Abundance of $\mathrm{C}^{13} \mathrm{O}_{2}{ }^{16}$ and $\mathrm{C}^{12} \mathrm{O}^{16} \mathrm{O}^{18}$}

As may be seen from Fig. 5, the positive branch of the $\mathrm{C}^{13} \mathrm{O}_{2}{ }^{16}$ band is overlapped by the negative branch of the $\mathrm{C}^{12} \mathrm{O}^{16} \mathrm{O}^{18}$ band and the negative branch of the former band

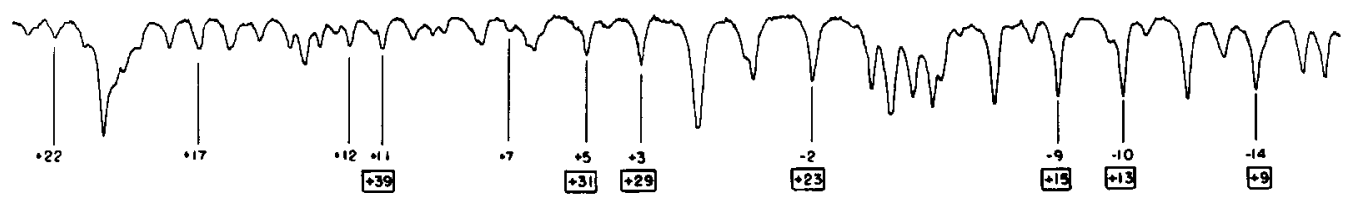

Fig. 5. Region of the $2 \cdot 05 \mu$ bands of $\mathrm{C}^{13} \mathrm{O}_{2}{ }^{16}$ and $\mathrm{C}^{12} \mathrm{O}^{16} \mathrm{O}^{18}$ in the solar spectrum. The serial numbers of the measured lines are given, the values inside the rectangles referring to $\mathrm{C}^{13} \mathrm{O}_{2}{ }^{16}$ and the others to $\mathrm{C}^{12} \mathrm{O}^{16} \mathrm{O}^{18}$

is hopelessly lost in the strong absorption by ordinary $\mathrm{CO}_{2}$. However, the weakness of the isotope lines suggests a method of analysis for blended pairs of lines in which the components are $\mathrm{C}^{13} \mathrm{O}_{2}{ }^{16}$ and $\mathrm{C}^{12} \mathrm{O}^{16} \mathrm{O}^{18}$.

Let $\tau_{1}$ be the absorption coefficient, as defined by equation (2), of a line of $\mathrm{C}^{13} \mathrm{O}_{2}{ }^{16}$ and $\tau_{2}$ that of a blended line of $\mathrm{C}^{12} \mathrm{O}^{16} \mathrm{O}^{18}$. If the lines in question are both very weak, the combined equivalent width will be equal to the sum of the absorption coefficients $\tau_{1}+\tau_{2}$. In the general case, the relation between the combined equivalent width and $\tau_{1}+\tau_{2}$ will depend upon the spacing of the components and upon the resolving power, and will be different for each pair of lines. The curves of growth will coincide in the linear portions, however, and in the intermediate portions will perhaps not 
deviate greatly from the curve of growth already determined for $\mathrm{CO}_{2}$, with $\gamma / 2 \pi$ $=1.7 \times 10^{9}$ sec. $^{-1}$.

The lines selected for measurement are given in Table 2. The first column gives the approximate wavelength, while the second and third columns give the serial numbers of the $\mathrm{C}^{13} \mathrm{O}_{2}{ }^{16}$ and $\mathrm{C}^{12} \mathrm{O}^{16} \mathrm{O}^{18}$ components, respectively. Four lines of the latter isotope are unblended. The equivalent widths of both blended and unblended lines were measured for a number of values of sec. $z$ in December, 1950, and converted to zenith values of $\tau_{2}$ and $\tau_{1}+\tau_{2}$ with the aid of the curve of growth in Fig. 3. By equation (2),

$$
\tau_{1}+\tau_{2}=\frac{\nu}{\nu_{0}} \frac{S_{0}}{Q_{r}}\left(2\left|m_{1}\right| N_{0_{1}} e^{-E_{J_{1}} / k T}+\left|m_{2}\right| N_{0_{2}} e^{-E_{J_{2}} / k T}\right),
$$

where $v_{0}$ is taken as equal for the two bands, and $N_{0_{1}}$ and $N_{0_{2}}$ are respectively the numbers of $\mathrm{C}^{13} \mathrm{O}_{2}{ }^{16}$ and $\mathrm{C}^{12} \mathrm{O}^{16} \mathrm{O}^{18}$ molecules per square centimetre through the zenith. For unblended lines, either one or the other of the two terms is set equal to zero. The factor 2 in the left-hand term takes account of the absence of alternate band lines in $\mathrm{C}^{13} \mathrm{O}_{2}{ }^{16}$. Average values of $\tau_{1}+\tau_{2}$ or of $\tau_{2}$ are given in the fourth column of Table 2. Each tabular entry is derived from at least ten separate measurements.

For each of the four unblended lines of $\mathrm{C}^{12} \mathrm{O}^{16} \mathrm{O}^{18}$, a value of $N_{0_{\mathrm{e}}}$ was calculated from equation (7), with the temperature assumed to be $240^{\circ} \mathrm{K}$. The values of $N_{0_{2}}$ are given in the fifth column of Table 2, the mean value being $1.53 \times 10^{19} \mathrm{~cm}^{-2}$. With this value of $N_{0_{2}}$, the remaining lines then gave the values of $N_{0_{1}}$ tabulated in the sixth column of Table 2. The mean is $3.54 \times 10^{19} \mathrm{~cm}^{-2}$. In centimetres N.T.P. reduced to sea level the abundances are $\mathrm{l} \cdot 54$ for $\mathrm{C}^{13} \mathrm{O}_{2}{ }^{16}$ and 0.67 for $\mathrm{C}^{12} \mathrm{O}^{16} \mathrm{O}^{18}$. The relative atomic abundances are therefore about 210 and 1000 for $\mathrm{C}_{12} / \mathrm{C}_{13}$ and $\mathrm{O}_{16} / \mathrm{O}_{18}$, respectively. Both ratios are about twice as large as precise determinations with the mass spectrograph (BIRGE, 1941). It seems more than likely, however, that the atmospheric abundances of isotopic $\mathrm{CO}_{2}$ are not abnormal and that the discrepancy can be attributed to uncertainties in the absorption coefficients, and to errors in the measurement and reduction of the isotope line intensities.*

To summarize, the average carbon dioxide content of the air above Mount Wilson, California, is $0 \cdot 040$ per cent. Any variation in abundance is less than about \pm 10 per cent and is probably within the error of the abundance determination. The abundances of the isotopic forms $\mathrm{C}^{13} \mathrm{O}_{2}{ }^{16}$ and $\mathrm{C}^{12} \mathrm{O}^{16} \mathrm{O}^{18}$ are found to be one-half as great as would be expected from mass spectrograph measurements of the abundances of $\mathrm{C}^{13}$ and $\mathrm{O}^{18}$, but at least part, if not all, of the discrepancy can be caused by errors in the absorption coefficients.

The writer wishes to acknowledge the valuable assistance of Dr. EDITH A. MüLLER, Mrs. Harriet Malitson, Miss Dilhan Ege, and Mr. John K. Wilkinson in the measurement and reduction of the tracings.

\section{REFERENCES}

BIRGE, R. T. . . . . . . . . . . . . 1941

Crawford, B. L. (Jr.) and Dinsmore, H. L. . . 1950

EgGers, D. F. (Jr.) and Crawford, B. L. . . . 195 I

Rev. Mod. Phys., 13, 223.

J. chem. Phys., 983, 1682 .

J. chem. Phys., 19, 1556.

* After this manuscript was sent to press, Dr. W. S. BENEDICT kindly supplied the writer with the results of his measurements of absorption coeffcients of isotope bands of $\mathrm{CO}_{2}$ at wave lengths longer than $2 \mu$. These results strongly suggest that the absorption coefficients of the isotope hands can differ greatly from those of the corresponding ordinary bands. 
GoldBerg, L. . . . . . . . . . . . . . 1951

Goldberg, L., Mohler, O. C. and Donovan, R. A. 1952

HALdANE, J. B. S. . . . . . . . . . 1936

HerzberG, G. . . . . . . . . . . . 1945

Howard, J. N. and Shaw, J. H. . . . . . 1952

HULST, H. C. VAN DE . . . . . . . . . . 1945

Locke, J. L. and HerzberG, L. . . . . . . 1953

McMath, R. R. and Mohler, O. C. . . . . 1949

Nielsen, A. H. and MigeotTe, M. . . . . . 1952

Penner, S. S. and Weber, D. . . . . . . . . 1951

Weber, D., Holm, R. J. and Penner, S. S. . . 1952

Wilson, E. B. (Jr.) and Wells, A. J. . . . . 1946
Ap. J., 113, 567.

J. Opt. Soc. Amer., 42, 1.

Nature (London), 137, 575.

Infra-red and Raman Spectra (Van

Nostrand, New York).

Phys. Rev., 87, 679.

Ann. Astrophys., 8 (1), 12.

Canad.J. Phys., 31, 504.

J. Opt. Soc. Amer., 39, 903.

Ann. Astrophys., 15, 134.

J. chem. Phys., 19, 1351.

J. chem. Phys., 20, 1820.

J. chem. Phys., 14, 578.

\title{
The Infra-red Spectrum of the Earth's Atmosphere
}

\author{
T. W. WORMELL \\ Cavendish Laboratory, Cambridge
}

SUMMARY

The results of the intensive study of the infra-red absorption spectrum of the Earth's atmosphere, which has proceeded during the last few years, are briefly reviewed and the potentialities of this method for determining the amount and the vertical distribution of rare polyatomic constituents are discussed.

\section{In'TRODUCTION}

THE predominant gases of the terrestrial atmosphere are nearly transparent over most of the frequency range in which resides the bulk of the energy, whether of the incoming solar beam or of the outward flux of terrestrial and atmospheric radiation, and the main absorption of energy, in either case, is due to the polyatomic constituents. These gases possess strong vibration and rotation absorption bands in the infra-red and nearly all the energy of temperature radiation from a source at ordinary atmospheric temperatures lies in the range of wavelengths between $3 \mu$ and $60 \mu$. In consequence, the polyatomic gases, although comprising a very small proportion of the whole atmosphere, are of overwhelming importance in determining its thermal state in so far as this is controlled by radiative transfer. Analysis of the effects of such transfer is extremely complicated due both to the complex nature of the absorption spectra, which are moreover pressure-dependent, and also to the different distributions in the atmosphere of the gases concerned. It is thus of importance from many points of view to have accurate knowledge of the identity, amount, and distribution of all polyatomic gases which are present in appreciable amount.

The study of the infra-red solar spectrum offers a very direct method of investigation, the more powerful in that the number and intensity of the lines of solar origin fall off rapidly for wavelengths exceeding about $2 \mu$ (GoLDBERG, 1950). The principal features of this spectrum, out to a wavelength of about $5 \mu$, had already been mapped by LANGLEY and АввотT before the end of the last century. A major advance had to await the great improvements, which have been made in the last fifteen years (SUTHERLAND and LEe, 1946-47), in the efficiency and speed of infra-red detectors. The use of photoconductive cells makes it possible to utilize nearly the full theoretical resolving power of a grating, for wavelengths out to about $3 \cdot 5 \mu$, and the limiting 